\title{
A NEW CLASS OF HOMOLOGY AND COHOMOLOGY 3-MANIFOLDS
}

\author{
D.J. GARITY, U.H. KARIMOV, D.D. REPOVŠ, AND F. SPAGGIARI
}

\begin{abstract}
We show that for any set of primes $\mathcal{P}$ there exists a space $M_{\mathcal{P}}$ which is a homology and cohomology 3 -manifold with coefficients in $\mathbb{Z}_{p}$ for $p \in \mathcal{P}$ and is not a homology or cohomology 3-manifold with coefficients in $\mathbb{Z}_{q}$ for $q \notin \mathcal{P}$. Moreover, $M_{\mathcal{P}}$ is neither a homology nor cohomology 3-manifold with coefficients in $\mathbb{Z}$ or $\mathbb{Q}$.
\end{abstract}

\section{INTRODUCTION}

In 1908 Tietze [7] constructed his famous 3-manifolds $L(p, q)$ called lens spaces. These spaces have many interesting properties. For example, lens spaces $L(5,1)$ and $L(5,2)$ have isomorphic fundamental groups and the same homology groups, but they do not have the same homotopy type (this was first proved in 1919 by Alexander [1]).

It is also well known that for every prime $q$, the lens space $M^{3}=L(q, 1)$ has the following properties:

- $M^{3}$ is a $\mathbb{Z}_{p}$-homology 3 -sphere for every prime $p \neq q$;

- $M^{3}$ is not a $\mathbb{Z}_{q}$-homology 3 -sphere; and

- $M^{3}$ is not a $\mathbb{Z}$-homology 3 -sphere.

We shall generalize this classical result as follows:

Theorem 1.1. Given any set of primes $\mathcal{P}$, there exists a 3-dimensional compact metric space $M_{\mathcal{P}}$ which has the following properties:

- $M_{\mathcal{P}}$ is a $\mathbb{Z}_{p}$-homology 3-manifold for every $p \in \mathcal{P}$;

- $M_{\mathcal{P}}$ is a $\mathbb{Z}_{p}$-cohomology 3-manifold for every $p \in \mathcal{P}$;

- $M_{\mathcal{P}}$ is not a $\mathbb{Z}_{q}$-homology 3-manifold for any $q \notin \mathcal{P}$;

- $M_{\mathcal{P}}$ is not a $\mathbb{Z}_{q}$-cohomology 3-manifold for any $q \notin \mathcal{P}$;

- $M_{\mathcal{P}}$ is not a $\mathbb{Z}$-homology 3-manifold;

- $M_{\mathcal{P}}$ is not a $\mathbb{Z}$-cohomology 3-manifold;

- $M_{\mathcal{P}}$ is not a $\mathbb{Q}$-homology 3-manifold; and

- $M_{\mathcal{P}}$ is not a $\mathbb{Q}$-cohomology 3-manifold.

\section{Preliminaries}

First, we fix the terminology, notation, and recall some well known facts. We let $L$ be the ring of integers $\mathbb{Z}$ or a field.

2010 Mathematics Subject Classification. Primary: 55P99, 57P05, 57P10; Secondary: 55N05, $55 \mathrm{~N} 35,57 \mathrm{M} 25$.

Key words and phrases. Cohomology 3-manifold, cohomological dimension, Borel-Moore homology, Čech cohomology, Milnor-Harlap exact sequence, lens space, ANR. 
Definition 2.1. (cf. [2, Corollary V.16.9]) A locally compact space $X$ is called a cohomology manifold of dimension $n$ over $L$ (denoted as $n-\mathrm{cm}_{L}$ ) if

(1) $X$ has finite cohomological dimension over $L\left(\operatorname{dim}_{\mathrm{L}} \mathrm{X}<\infty\right)$;

(2) $X$ is cohomologically locally connected over $L\left(\operatorname{clc}_{L}^{\infty}\right)$; and

(3) for all $x \in X$,

$$
\check{H}^{p}(X, X-\{x\} ; L) \cong\left\{\begin{array}{lll}
L & \text { for } & p=n \\
0 & \text { for } & p \neq n
\end{array}\right.
$$

where $\check{H}^{*}$ are $\breve{C}$ ech cohomology groups with coefficients in $L$.

Definition 2.2. A locally compact space $X$ is called a homology manifold of dimension $n$ over $L$ (denoted as $\left.n-h m_{L}\right)$ if

(1) $X$ has finite cohomological dimension over $L\left(\operatorname{dim}_{\mathrm{L}} \mathrm{X}<\infty\right)$;

(2) for all $x \in X$,

$$
H_{p}(X, X-\{x\} ; L) \cong\left\{\begin{array}{lll}
L & \text { for } & p=n \\
0 & \text { for } & p \neq n
\end{array}\right.
$$

where $H_{*}$ are Borel-Moore homology groups with coefficients in $L$.

Homology manifolds will stand for homology $\mathbb{Z}$-manifolds.

Any $n$-dimensional cohomology manifold $\left(n-c m_{L}\right)$ is also an $n$-dimensional homology manifold $\left(n-h m_{L}\right)$ by [2, Theorem V.16.8]. Therefore we will construct only cohomology manifolds which will be automatically homology manifolds by this theorem.

Example 2.3. For the construction and some simple properties of lens spaces see $[5,6]$. In particular, the homology groups of the lens space $M^{3}=L(q, 1)$ are

$$
H_{n}\left(M^{3} ; \mathbb{Z}\right)= \begin{cases}\mathbb{Z} & n=0,3 \\ \mathbb{Z}_{q} & n=1 \\ 0 & n=2 \text { or } n \geq 4\end{cases}
$$

By the Universal Coefficients Theorem, we have for any abelian group $G$,

$$
H_{n}\left(M^{3} ; G\right) \cong H_{n}\left(M^{3} ; \mathbb{Z}\right) \otimes G \oplus H_{n-1}\left(M^{3} ; \mathbb{Z}\right) * G
$$

Therefore, if $p$ and $q$ are prime and $p \neq q$ then

$$
H_{n}\left(M^{3} ; \mathbb{Z}_{p}\right) \cong \begin{cases}\mathbb{Z}_{p} & \text { if } n=0,3 \\ 0 & \text { otherwise }\end{cases}
$$

whereas

$$
H_{n}\left(M^{3} ; \mathbb{Z}_{q}\right) \cong \begin{cases}\mathbb{Z}_{q} & n=0,1,3 \\ 0 & \text { otherwise }\end{cases}
$$

Consider now the suspension $N^{4}=\Sigma M^{3}$ of $M^{3}$. Local conditions of Definitions 2.1 and 2.2 are clearly satisfied for $N^{4}$ since $M^{3}$ is a 3-manifold. Therefore $N^{4}$ is a $4-h m_{\mathbb{Z}_{p}}$ and a $4-c m_{\mathbb{Z}_{p}}$. However, $N^{4}$ is neither a $4-h m_{\mathbb{Z}_{q}}$ nor a $4-c m_{\mathbb{Z}_{q}}$ if $p$ and $q$ are prime and $p \neq q$ (cf. [2]). 


\section{Proof of Theorem 1.1}

Let $\mathcal{P}=\left\{p_{i}\right\}_{i \in K}$, for $K=\mathbb{N}$ or $K=\{1, \ldots, k\}$, be a set of some prime numbers. If the set $K$ is infinite then we define the numbers $n_{i}$ as $n_{i}=p_{1} \cdot p_{2} \cdot p_{3} \cdots p_{i}$. If the set $K$ is finite and consists of exactly $k$ elements, then define $n_{i}$ as $n_{i}=p_{1} \cdot p_{2} \cdot p_{3} \cdots p_{k}$ for all $i$.

Let $X$ be a solenoid in the 3-dimensional sphere $S^{3}$, i.e. the inverse limit of solid tori corresponding to the following inverse system:

$$
\mathbb{Z} \stackrel{n_{1}}{\longleftarrow} \mathbb{Z} \stackrel{n_{2}}{\longleftarrow} \mathbb{Z} \stackrel{n_{3}}{\longleftarrow} \ldots
$$

naturally embedded in $S^{3}$, see e.g. [3, Chapter IX, Exercise E.4].

Let us prove that the quotient space $S^{3} / X$ is a cohomology 3 -manifold $c m_{\mathbb{Z}_{p}}$. It is obvious that $S^{3} / X$ is 3 -dimensional, compact and metrizable. So the space $S^{3} / X$ satisfies the condition (1) of Definition 2.1.

To prove that the space $S^{3} / X$ satisfies the conditions (2) and (3) of Definition 2.1, let's calculate first the groups $\breve{H}^{n}\left(S^{3} / X,\{x\} ; G\right)$ with respect to the one-point subset $\{x\}=X / X$ for $G \cong \mathbb{Z}_{p}, p \in \mathcal{P} ; G \cong \mathbb{Z}_{q}, q \notin \mathcal{P} ; G \cong \mathbb{Z} ; G \cong \mathbb{Q}$. Since $S^{3} / X$ is connected and 3 -dimensional it follows that

$$
\check{H}^{0}\left(S^{3} / X,\{x\} ; G\right) \cong 0 \text { and } \check{H}^{n}\left(S^{3} / X,\{x\} ; G\right) \cong 0 \text { for } n>3 .
$$

Let $U_{i}$ be the open $i$-th solid torus neighborhood of $X$ in $S^{3}$ (see [3]). Then $\left\{U_{i} / X\right\}_{i \in \mathbb{N}}$ is a neighborhood base of $x$ in $S^{3} / X$. By continuity of the Cech cohomology and by the Excision Axiom it follows that:

$$
\begin{aligned}
& \check{H}^{n}\left(S^{3} / X,\{x\} ; G\right) \cong \check{H}^{n}\left(\lim _{\leftarrow} S^{3} / \bar{U}_{i}, \bar{U}_{i} / \bar{U}_{i} ; G\right) \cong \\
& \cong \lim _{\rightarrow} \check{H}^{n}\left(S^{3} / \bar{U}_{i}, \bar{U}_{i} / \bar{U}_{i} ; G\right) \cong \lim _{\rightarrow} \check{H}^{n}\left(S^{3}, \bar{U}_{i} ; G\right) .
\end{aligned}
$$

For $n=1$ we have the exact sequence of the pair $\left(S^{3}, \overline{U_{i}}\right)$ :

$$
\check{H}^{0}\left(S^{3} ; G\right) \longrightarrow \check{H}^{0}\left(\overline{U_{i}} ; G\right) \longrightarrow \check{H}^{1}\left(S^{3}, \bar{U}_{i} ; G\right) \longrightarrow \check{H}^{1}\left(S^{3}, G\right) \longrightarrow \check{H}^{1}\left(\overline{U_{i}} ; G\right) .
$$

Since the 1-dimensional cohomology of the 3 -sphere is trivial for any group of coefficients $G$ and $\overline{U_{i}}$ is connected space for every $i$, it follows that $\check{H}^{1}\left(S^{3}, \bar{U}_{i} ; G\right) \cong$ 0 , therefore $\check{H}^{n}\left(S^{3} / X,\{x\} ; G\right) \cong 0$ and, in particular,

$$
\check{H}^{1}\left(S^{3} / X,\{x\} ; \mathbb{Z}_{p}\right) \cong 0
$$

and

$$
\check{H}^{1}\left(S^{3} / X,\{x\} ; \mathbb{Z}_{q}\right) \cong 0, \check{H}^{1}\left(S^{3} / X,\{x\} ; \mathbb{Z}\right) \cong 0, \check{H}^{1}\left(S^{3} / X,\{x\} ; \mathbb{Q}\right) \cong 0 .
$$

For $n=2$ we have the following exact sequence of the pair $\left(S^{3}, \overline{U_{i}}\right)$ :

$$
\check{H}^{1}\left(S^{3} ; G\right) \longrightarrow \check{H}^{1}\left(\bar{U}_{i} ; G\right) \longrightarrow \check{H}^{2}\left(S^{3}, \bar{U}_{i} ; G\right) \longrightarrow \check{H}^{2}\left(S^{3}, G\right) \longrightarrow \check{H}^{2}\left(\overline{U_{i}} ; G\right) .
$$

The cohomology groups $\check{H}^{1}\left(S^{3} ; G\right)$ and $\check{H}^{2}\left(S^{3}, G\right)$ are trivial, and the groups $\check{H}^{1}\left(\bar{U}_{i} ; G\right)$ are isomorphic to $G$ since $U_{i}$ has the homotopy type of a circle. The homomorphisms $\check{H}^{1}\left(\bar{U}_{i} ; G\right) \rightarrow \check{H}^{1}\left(\bar{U}_{i+1} ; G\right)$ are multiplications by $n_{i}$ that take the group $G$ into itself.

Therefore for the group of coefficients $G \cong \mathbb{Z}_{p}$ it follows that

$$
\check{H}^{2}\left(S^{3} / X,\{x\} ; \mathbb{Z}_{p}\right) \cong 0 .
$$


However,

$$
\check{H}^{2}\left(S^{3} / X,\{x\} ; \mathbb{Z}_{q}\right) \nsucceq 0, \check{H}^{2}\left(S^{3} / X,\{x\} ; \mathbb{Z}\right) \nsucceq 0, \check{H}^{2}\left(S^{3} / X,\{x\} ; \mathbb{Q}\right) \nsucceq 0 .
$$

For $n=3$ consider the following cohomology exact sequence for the pair $\left(S^{3}, \overline{U_{i}}\right)$ :

$$
\check{H}^{2}\left(\bar{U}_{i} ; G\right) \longrightarrow \check{H}^{3}\left(S^{3}, \bar{U}_{i} ; G\right) \longrightarrow \check{H}^{3}\left(S^{3}, G\right) \longrightarrow \check{H}^{3}\left(\bar{U}_{i} ; G\right) .
$$

Since $\bar{U}_{i} \simeq S^{1}$, it follows that:

$$
\check{H}^{3}\left(S^{3} / X, x ; G\right) \cong G .
$$

Let us calculate the groups $\check{H}^{n}\left(S^{3} / X-\{x\} ; \mathbb{Z}_{p}\right)$. The space $S^{3} / X-\{x\}$ is the union $\bigcup_{i=1}^{\infty}\left(S^{3}-U_{i}\right)$ of an increasing sequence of "complementary" solid tori.

For $n=1$ we have the following exact sequence of Milnor-Harlap [4, Theorem $1]$ :

$$
0 \rightarrow \lim ^{(1)} \check{H}^{0}\left(S^{3}-U_{i} ; \mathbb{Z}_{p}\right) \rightarrow \check{H}^{1}\left(S^{3}-X ; \mathbb{Z}_{p}\right) \rightarrow \lim _{\longleftarrow} \check{H}^{1}\left(S^{3}-U_{i} ; \mathbb{Z}_{p}\right) \rightarrow 0,
$$

where $\lim ^{(1)}$ is the first derived functor of the functor of the inverse limit. Since $p \in \mathcal{P}$ it follows that the inverse limit $\lim \check{H}^{1}\left(S^{3}-U_{i} ; \mathbb{Z}_{p}\right)$ is trivial. The group $\lim ^{(1)} \check{H}^{0}\left(S^{3}-U_{i} ; \mathbb{Z}_{p}\right)$ is trivial since the corresponding inverse sequence satisfies the Mittag-Leffler (ML) condition, so we have

$$
\check{H}^{1}\left(S^{3}-X ; \mathbb{Z}_{p}\right) \cong 0
$$

Analogously, it is easy to see that

$$
\check{H}^{1}\left(S^{3}-X ; \mathbb{Z}_{q}\right) \nsucceq 0 \text { for } q \notin \mathcal{P}, \quad \check{H}^{1}\left(S^{3}-X ; \mathbb{Z}\right) \cong 0, \check{H}^{1}\left(S^{3}-X ; \mathbb{Q}\right) \nsucceq 0 .
$$

Let $n=2$, then we have the Milnor-Harlap exact sequence for the presentation $S^{3} / X-\{x\}=\bigcup_{i=1}^{\infty}\left(S^{3}-U_{i}\right)$ :

$$
0 \rightarrow \lim ^{(1)} \check{H}^{1}\left(S^{3}-U_{i} ; \mathbb{Z}_{p}\right) \rightarrow \check{H}^{2}\left(S^{3}-X ; \mathbb{Z}_{p}\right) \rightarrow \underset{\longleftarrow}{\lim } \check{H}^{2}\left(S^{3}-U_{i} ; \mathbb{Z}_{p}\right) \rightarrow 0 .
$$

The groups $\lim ^{(1)} \check{H}^{1}\left(S^{3}-U_{i} ; \mathbb{Z}_{p}\right)$ are trivial since the groups $\check{H}^{1}\left(S^{3}-U_{i} ; \mathbb{Z}_{p}\right)$ are isomorphic to the finite group $\mathbb{Z}_{p}$ and the corresponding inverse sequence satisfies the ML condition. The groups $\check{H}^{2}\left(S^{3}-U_{i} ; \mathbb{Z}_{p}\right)$ are also trivial since the "complementary" solid tori have the homotopy type of the circle. Therefore

$$
\check{H}^{2}\left(S^{3}-X ; \mathbb{Z}_{p}\right) \cong 0 .
$$

For $n=3$ we have the exact sequence of Milnor-Harlap for the same presentation of $S^{3} / X-\{x\}$ as before:

$$
0 \rightarrow \lim ^{(1)} \check{H}^{2}\left(S^{3}-U_{i} ; \mathbb{Z}_{p}\right) \rightarrow \check{H}^{3}\left(S^{3}-X ; \mathbb{Z}_{p}\right) \rightarrow \underset{\lim }{\check{H}^{3}}\left(S^{3}-U_{i} ; \mathbb{Z}_{p}\right) \rightarrow 0 .
$$

The groups $\check{H}^{3}\left(S^{3}-U_{i} ; \mathbb{Z}_{p}\right)$ and $\check{H}^{2}\left(S^{3}-U_{i} ; \mathbb{Z}_{p}\right)$ are trivial since the spaces $S^{3}-U_{i}$ have the homotopy type of a circle. Therefore:

$$
\check{H}^{3}\left(S^{3}-X ; \mathbb{Z}_{p}\right) \cong 0 \text {. }
$$

Next, let us calculate the groups $\check{H}^{n}\left(S^{3} / X, S^{3} / X-X / X ; G\right)$ for certain groups $G$.

Since the space $S^{3} / X$ is connected and $\operatorname{dim} S^{3} / X=3$ it follows that these groups are trivial groups for $n=0, n>3$. 
Since the space $S^{3}-X$ is connected and $\check{H}^{1}\left(S^{3} / X ; \mathbb{Z}_{p}\right) \cong 0$ by (2), it follows by the exact cohomology sequence of the pair $\left(S^{3} / X, S^{3} / X-X / X\right)$ (or the pair $\left(S^{3} / X, S^{3}-X\right)$ since $\left.S^{3} / X-X / X \cong S^{3}-X\right)$ that

$$
\check{H}^{1}\left(S^{3} / X, S^{3}-X ; \mathbb{Z}_{p}\right) \cong 0
$$

By the exact sequence:

$\check{H}^{1}\left(S^{3}-X ; \mathbb{Z}_{p}\right) \stackrel{\delta}{\longrightarrow} \check{H}^{2}\left(S^{3} / X, S^{3}-X ; \mathbb{Z}_{p}\right) \longrightarrow \check{H}^{2}\left(S^{3} / X ; \mathbb{Z}_{p}\right) \longrightarrow \check{H}^{2}\left(S^{3}-X ; \mathbb{Z}_{p}\right)$ and since the groups $\check{H}^{1}\left(S^{3}-X ; \mathbb{Z}_{p}\right)$ and $\check{H}^{2}\left(S^{3} / X ; \mathbb{Z}_{p}\right)$ are trivial by $(7)$ and $(4)$ it follows that

$$
\check{H}^{2}\left(S^{3} / X, S^{3}-X ; \mathbb{Z}_{p}\right) \cong 0 .
$$

For any group of coefficients the corresponding homomorphism $\delta$ is a monomorphism by (3). Since the groups $\check{H}^{1}\left(S^{3}-X ; \mathbb{Z}_{q}\right)$ for $q \notin \mathcal{P}, \check{H}^{1}\left(S^{3}-X ; Q\right)$ are nontrivial by (8), and the groups $\check{H}^{1}\left(S^{3} / X ; \mathbb{Z}_{q}\right), \check{H}^{1}\left(S^{3} / X ; Q\right)$ are trivial, it follows that

$$
\check{H}^{2}\left(S^{3} / X, S^{3}-X ; \mathbb{Z}_{q}\right) \supsetneqq 0, \check{H}^{2}\left(S^{3} / X, S^{3}-X ; \mathbb{Q}\right) \nsucceq 0 .
$$

Consider the following exact sequence of the pair $\left(S^{3} / X, S^{3}-X ; \mathbb{Z}_{p}\right)$ :

$\check{H}^{2}\left(S^{3}-X ; \mathbb{Z}_{p}\right) \stackrel{\delta}{\longrightarrow} \check{H}^{3}\left(S^{3} / X, S^{3}-X ; \mathbb{Z}_{p}\right) \longrightarrow \check{H}^{3}\left(S^{3} / X ; \mathbb{Z}_{p}\right) \longrightarrow \check{H}^{3}\left(S^{3}-X ; \mathbb{Z}_{p}\right)$. The groups $\check{H}^{2}\left(S^{3}-X ; \mathbb{Z}_{p}\right)$ and $\check{H}^{3}\left(S^{3}-X ; \mathbb{Z}_{p}\right)$ are trivial by (9) and (10), respectively. Next, observe that $\check{H}^{3}\left(S^{3} / X ; \mathbb{Z}_{p}\right) \cong \mathbb{Z}_{p}$. Therefore

$$
\check{H}^{3}\left(S^{3} / X, S^{3}-X ; \mathbb{Z}_{p}\right) \cong \mathbb{Z}_{p} \text {. }
$$

Let us show that $S^{3} / X$ is $c l c_{Z_{p}}^{\infty}$ at all points. Obviously, the space $S^{3} / X$ is a $c l c_{Z_{p}}^{\infty}$ space for all points except at the point $x=X / X$ since $S^{3}-X$ is an open manifold. As mentioned before, the sets $\left\{U_{i} / X\right\}_{i \in \mathbb{N}}$ form a neighborhood base of the point $x$. Consider the groups $\check{H}^{n}\left(U_{i} / X, X / X ; \mathbb{Z}_{p}\right)$. By the Excision Axiom it follows that $\check{H}^{n}\left(U_{i} / X, X / X ; \mathbb{Z}_{p}\right) \cong \check{H}^{n}\left(U_{i}, X ; \mathbb{Z}_{p}\right)$.

From the following commutative diagram with exact rows

$$
\begin{array}{lllll}
0 \cong \check{H}^{0}\left(X ; \mathbb{Z}_{p}\right) & \longrightarrow & \check{H}^{1}\left(U_{i}, X ; \mathbb{Z}_{p}\right) & \longrightarrow \check{H}^{1}\left(U_{i} ; \mathbb{Z}_{p}\right) & \cong \mathbb{Z}_{p} \\
\downarrow & \downarrow \pi^{i} & & \\
0 \cong \check{H}^{0}\left(X ; \mathbb{Z}_{p}\right) & \longrightarrow & \check{H}^{1}\left(U_{i+1}, X ; \mathbb{Z}_{p}\right) & \longrightarrow \check{H}^{1}\left(U_{i+1} ; \mathbb{Z}_{p}\right) & \cong \mathbb{Z}_{p}
\end{array}
$$

it follows that for a large enough $i$, the homomorphism

$$
\check{H}^{1}\left(U_{i}, X ; \mathbb{Z}_{p}\right) \stackrel{\pi^{i}}{\longrightarrow} \check{H}^{1}\left(U_{i+1}, X ; \mathbb{Z}_{p}\right)
$$

is trivial. Therefore

$$
S^{3} / X \text { is a } 1-\operatorname{clc}_{\mathbb{Z}_{p}} \text { space. }
$$

By the analogous diagram for the group of coefficients $\mathbb{Z}$ it is easy to see that the homomorphism $\check{H}^{1}\left(U_{i}, X ; \mathbb{Z}\right) \stackrel{\pi^{i}}{\longrightarrow} \check{H}^{1}\left(U_{i+1}, X ; \mathbb{Z}\right)$ is a monomorphism of the group $\mathbb{Z}$. Therefore

$$
S^{3} / X \text { is not } 1-c l c_{\mathbb{Z}}
$$

By the exact sequence

$$
\check{H}^{1}\left(X ; \mathbb{Z}_{p}\right) \longrightarrow \check{H}^{2}\left(U_{i}, X ; \mathbb{Z}_{p}\right) \longrightarrow \check{H}^{2}\left(U_{i}, \mathbb{Z}_{p}\right)
$$


since $\check{H}^{2}\left(U_{i}, \mathbb{Z}_{p}\right) \cong 0$ and the Čech cohomology group $\check{H}^{1}\left(X ; \mathbb{Z}_{p}\right)$ is obviously isomorphic to the direct limit of the sequence

$$
\mathbb{Z}_{p} \stackrel{\times n_{1}}{\rightarrow} \mathbb{Z}_{p} \stackrel{\times n_{2}}{\rightarrow} \mathbb{Z}_{p} \stackrel{\times n_{3}}{\rightarrow} \cdots
$$

it follows that $\check{H}^{2}\left(U_{i}, X ; \mathbb{Z}_{p}\right) \cong 0$. By the Excision Axiom it follows that

$$
\check{H}^{2}\left(U_{i} / X, X / X ; \mathbb{Z}_{p}\right) \cong 0
$$

and

$$
S^{3} / X \text { is a } 2-\operatorname{clc}_{\mathbb{Z}_{p}} \text { space. }
$$

By the following exact sequence of the pair $\left(U_{i}, X\right)$

$$
\check{H}^{2}\left(X ; \mathbb{Z}_{p}\right) \longrightarrow \check{H}^{3}\left(U_{i}, X ; \mathbb{Z}_{p}\right) \longrightarrow \check{H}^{3}\left(U_{i}, \mathbb{Z}_{p}\right)
$$

and since the space $X$ is 1 -dimensional and $U_{i}$ is homotopy equivalent to the circle it follows that $\check{H}^{3}\left(U_{i}, X ; \mathbb{Z}_{p}\right) \cong 0$ therefore $\check{H}^{3}\left(U_{i} / X, X / X ; \mathbb{Z}_{p}\right) \cong 0$ and

$$
S^{3} / X \text { is a } 3-\operatorname{clc}_{\mathbb{Z}_{p}} \text { space. }
$$

By the local connectedness of the space $S^{3} / X$, by (15), (17), (18) and since $\operatorname{dim} S^{3} / X=3$ it follows that $S^{3} / X$ is a $c l c_{\mathbb{Z}_{p}}^{\infty}$ space and $S^{3} / X$ satisfies the condition (2) of Definition 2.1.

It follows by (11), (12), and (14) that $S^{3} / X$ satisfies the condition (3) of Definition 2.1 , therefore $S^{3} / X$ is a $3-c m_{\mathbb{Z}_{p}}$ and a $3-h m_{\mathbb{Z}_{p}}$.

However, the space $S^{3} / X$ is neither a $3-c m_{\mathbb{Z}_{q}}$ nor a $3-c m_{\mathbb{Q}}$ since $\check{H}^{2}\left(S^{3} / X, S^{3}-\right.$ $\left.X ; \mathbb{Z}_{q}\right) \varsubsetneqq 0$ and $\check{H}^{2}\left(S^{3} / X, S^{3}-X ; \mathbb{Q}\right) \nsucceq 0$ by $(16)$, and is not a $3-c m_{\mathbb{Z}}$ since it is not a $1-c c_{\mathbb{Z}}$. This completes the proof.

\section{EPilogue}

The spaces which we have constructed are not ANR's, so the following remains an open question:

Question 4.1. Let $\mathcal{P}$ be any set of prime numbers. Does there exist a 3-dimensional $A N R X_{\mathcal{P}}$ with the following properties:

(1) $X_{\mathcal{P}}$ is a $\mathbb{Z}_{p}$-homology 3-manifold for every $p \in \mathcal{P}$; and

(2) $X_{\mathcal{P}}$ is not a $\mathbb{Z}_{q}$-homology 3-manifold for any $q \notin \mathcal{P}$ ?

\section{AcKnowledgements}

This research was supported by the Slovenian Research Agency grants No. BIUS/13-14-027, P1-0292, J1-4144, J1-5435, and J1-6721. We thank Allen Hatcher for noticing an inaccuracy in the initial version of Example 2.3.

\section{REFERENCES}

[1] J. W. Alexander, Note on two three-dimensional manifolds with the same group, Trans Amer. Math. Soc. 20 (1919), 339-342.

[2] G. E. Bredon, Sheaf Theory, 2nd Ed., Graduate Texts in Math. No. 170, Springer, Berlin, 1997.

[3] S. Eilenberg, N. Steenrod, Foundations of Algebraic Topology, Princeton University Press, Princeton, N. J., 1952. 
[4] A. È. Harlap, Local homology and cohomology, homology dimension and generalized manifolds, Math. Sb. (N. S.) 96 (138) (1975), 347-373. (in Russian); English transl.: Math. USSR-Sb. 25:3 (1975), 323-349.

[5] A. Hatcher, Algebraic Topology, Cambridge Univ. Press, Cambridge, 2002.

[6] H. Seifert, W. Threlfall, A Textbook of Topology, Academic Press, New York, 1980.

[7] H. Tietze, Uber die topologischen Invarianten mehrdimensionaler Mannigfaltigkeiten, Monathsh. Für Math. Und Phys. 19 (1908), 1-118.

Department of Mathematics, Oregon State University, Corvallis, Oregon, USA

E-mail address: garity@math.oregonstate.edu

Institute of Mathematics, Academy of Sciences of Tajikistan, Dushanbe, Tajikistan

E-mail address: umedkarimov@gmail.com

Faculty of Education and Faculty of Mathematics and Physics, University of Ljubljana \& Institute of Mathematics, Physics and Mechanics, Ljubljana, Slovenia

E-mail address: dusan.repovs@guest.arnes.si

Department of Mathematics, University of Modena and Reggio Emilia, Modena, Italy

E-mail address: fulvia.spaggiari@unimore.it 